\title{
Snuffbox to the rescue: distal transradial approach for cardiac catheterisation after failed ipsilateral radial puncture
}

\author{
Eleftherios Kontopodis, Aggeliki Rigatou, loannis Tsiafoutis, Efstathios Lazaris, Michael Koutouzis \\ Department of Cardiology, Hellenic Red Cross Hospital of Athens, Athens, Greece
}

We demonstrate a case of cardiac catheterisation through the left distal radial artery (dRA), in a patient with end-stage renal disease and an ipsilateral non-functioning arteriovenous (AV) fistula. An 83-year-old man with end-stage renal disease was referred for coronary angiography due to stable angina (Canadian Cardiovascular Society class III). The use of the right forearm as an access site for cardiac catheterisation was contraindicated due to the presence of a functioning AV fistula used for the haemodialysis. Moreover, transfemoral approach was not feasible because the patient had non-palpable femoral arteries and reported symptoms of intermittent claudication. We considered the left forearm approach, despite the existence of an ipsilateral non-functioning AV fistula (Fig. 1A), and the left radial artery was chosen as the primary access point. However, the puncture was unsuccessful, resulting in a localised haematoma and local loss of the radial pulse. The ipsilateral ulnar artery was non-palpable; therefore, no attempt of the ulnar artery puncture was carried out. The left dRA, located in the anatomical snuffbox, was chosen as an alternative access site (Fig. 1A). The puncture was successfully performed, and we introduced a 6-F, 11-cm-long hydrophilic sheath (St. Jude Medical Inc., Saint Paul, MN, USA). The arm angiogram depicted the non-functioning AV fistula (Fig. 1B) at the level of the brachial artery. Coronary angiography (CAG) was successfully performed. An $80 \%$ stenosis of the left anterior descending artery was identified and successfully treated with a $2.75 \times 26 \mathrm{~mm}$ zotarolimus eluting stent (Resolute Integrity, Medtronic Inc., Minneapolis, MN, USA). At the end of the procedure the sheath was removed, and haemostasis was achieved through manual compression. A bandage was used for $3 \mathrm{~h}$ (Fig. 1C). Transradial approach is regarded as the default access for coronary catheterisation and is associated with a lower rate of access-related bleeding events compared with the femoral approach [1]. The benefits of the transradial approach are even more pronounced in high-risk populations, such as in patients with end-stage renal disease [2]. Access through the dRA in the anatomical snuffbox has recently been introduced for cardiac catheterisation, and it seems to be a feasible and safe approach [3]. An algorithm promoting the use of the forearm (vs. femoral) approach during cardiac catheterisation has already been demonstrated, evaluating the possibility of using all four (two radial and two ulnar) wrist arteries [4]. In our case the left dRA was used for CAG and angioplasty after a failed puncture of the ipsilateral radial artery. The use of dRAs as access sites may increase the number of possible forearm access routes from four to six (two radial, two ulnar, and two distal radial arteries) and further reduce the use of femoral approach for cardiac catheterisation. Distal radial artery access for CAG is a new promising technique, which seems to be gaining popularity lately. As demonstrated in our case, dRA can be a safe and feasible alternative access site after the failure of the traditional forearm approach. Further randomised trials are needed in order to evaluate the possible benefits of this approach.

\section{References}

1. Hamon M, Pristipino C, Di Mario C, et al. Position paper by the European Association of Percutaneous Cardiovascular Interventions and Working Groups on Acute Cardiac Care and Thrombosis of the European Society of Cardiology. EuroIntervention. 2013; 8(11): 1242-1251, doi: 10.4244/EIJV8I11A192, indexed in Pubmed: 23354100.

2. Koutouzis M, Sfyroeras GS, Maniotis C, et al. Forearm versus femoral approach for cardiac catheterization in end-stage renal disease patients. J Invasive Cardiol. 2018; 30(3): 110-114, indexed in Pubmed: 29493512.

3. Kiemeneij F. Left distal transradial access in the anatomical snuffbox for coronary angiography (ldTRA) and interventions (IdTRI). EuroIntervention. 2017; 13(7): 851-857, doi: 10.4244/EIJ-D-17-00079, indexed in Pubmed: 28506941.

4. Gatzopoulos D, Agelaki M, Rigatou A, et al. Alternative access site choice after initial radial access site failure for coronary angiography and intervention. Journal of Geriatric Cardiology. 2018 (In press).

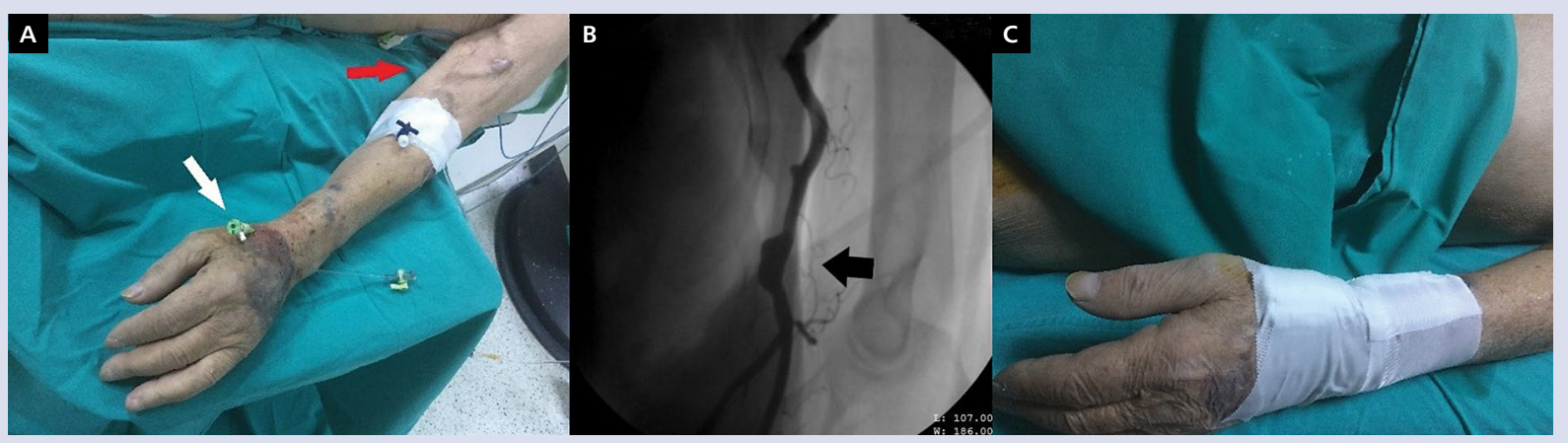

Figure 1. A. Left forearm after catheterisation: a sheath in the left distal radial artery (dRA) (white arrow), non-functioning arteriovenous (AV) fistula (red arrow); B. Angiogram after the injection of a contrast agent through the left dRA: non-functioning AV fistula (black arrow) and free access through the left radial and branchial arteries; $\mathbf{C}$. Left forearm: bandage after manual compression

\section{Address for correspondence:}

Dr. Eleftherios Kontopodis, $2^{\text {nd }}$ Department of Cardiology, Hellenic Red Cross Hospital, Athanasaki 1, GR 11526, Athens, Greece, tel: +306977179554,

e-mail: ekontopodis@gmail.com

Conflict of interest: none declared

Kardiologia Polska Copyright (c) Polish Cardiac Society 2018 